\title{
Studies on electrical and thermal properties of dental glass ionomer cement
}

\author{
Tadiboyina Anil Babu ${ }^{1}$, Kocharlakota Venkata Ramesh ${ }^{2}$, Devulapalli Lakshmipathi Sastry ${ }^{1^{*}}$ \\ ${ }^{1}$ Department of Physics, Andhra University, Visakhapatnam, India \\ ${ }^{2}$ Department of Physics, GITAM Institute of Technology (GIT), GITAM University, Visakhapatnam, India \\ Email: *sastry_dl@yahoo.co.in
}

Received 5 September 2012; revised 5 October 2012; accepted 20 October 2012

\begin{abstract}
A commercially available dental Glass Ionomer Cement (GIC) was studied after setting at room temperature $(300 \mathrm{~K})$ to understand its DC electrical conductivity, dielectric and thermal properties. The dental GIC's are supposed to have free mobile charge carriers like $\mathrm{F}^{-}$ions. Interestingly this material loses its conductivity above $80^{\circ} \mathrm{C}$ and behaves like a nonpolar substance. The frequency dependent dielectric studies also indicate the loss of mobile charge carriers in the samples annealed at $80^{\circ} \mathrm{C}$. The DSC and TGA studies indicate that the material loses $\mathrm{H}_{2} \mathrm{O}$ exothermically at $100^{\circ} \mathrm{C}$. This is attributed to the onset of a secondary setting reaction.
\end{abstract}

Keywords: Dental Glass Ionomer Cement; Dielectric Properties; DSC Studies; DC Conductivity

Measurements

\section{INTRODUCTION}

Glass Ionomer Cements (GICs) are formed by the acid base reaction of an aqueous polymeric acid with an alumino silicate glass powder [1]. These glass ionomer cements find extensive use as dental restoratives for their biocompatibility, favorable mechanical properties and also because of sustained fluoride ion release which is suitable for antibacterial activity of these materials [2]. Many studies were carried out to study the relationship between stoichiometry and fluorine ion release of these GIC materials [3] which indicate prolonged fluorine ion release in vitro or in vivo. Several studies using SEM [4], IR [5] and Raman spectroscopies [6] as well as solid state high resolution NMR studies [7] are reported on these materials to study the relationship between structure, composition, setting reaction and the release of fluorine and other ions like $\mathrm{Al}^{3+}$. But so far no report has been found for the temperature dependent ionic conduc-

"Corresponding author. tivity of these materials in the solid state. The measurement of dielectric constant and resistivity of these materials has been used to monitor their setting characteristics $[8,9]$.

We studied the temperature dependent DC electrical conductivity of a glass ionomer cement after allowing it to set for nearly 48 hours. Its dielectric and thermal properties were also studied. The results obtained were found to be interesting and are reported here.

\section{EXPERIMENTAL}

A commercially available Glass Ionomer Cement (Shanghai Medical Instruments Co. Ltd) has been chosen for the present investigations. The glass powder and the polymeric liquid are mixed according to the manufacturer's instructions. The thoroughly mixed paste has been transferred to a stainless steel die with 9-mm diameter holes and pressed into pellets of 2-mm or 3-mm diameter. The cement was allowed to set for more than 48 hours at room temperature $(300 \mathrm{~K})$ in a dry atmosphere under mild pressure. The pellets used for the study of electrical properties were coated with a thin layer of silver electroding on either sides of the pellet.

These pellets are ground into a fine powder for X-ray, DSC and TGA studies.

$\mathrm{X}$-ray diffractograms were recorded using a Seifert X-ray diffractometer. A Perkin Elmer DSC-7 instrument was used for recording DSC and TGA thermograms. The conductivity of the sample was calculated by measuring the resistivity of the sample using a Keithley (model 614) digital electrometer. The capacitance and loss factor were measured using a Hioki 3532-50 LCR meter. All samples were stored in a dessicator containing silica gel.

\section{RESULTS AND DISCUSSION}

The X-ray diffractograms of the as prepared GIC pellet and the starting glass powder are presented in Figures 1(a)-(b). The X-ray diffractograms of the glass powders are featureless and are indicative of highly disordered 


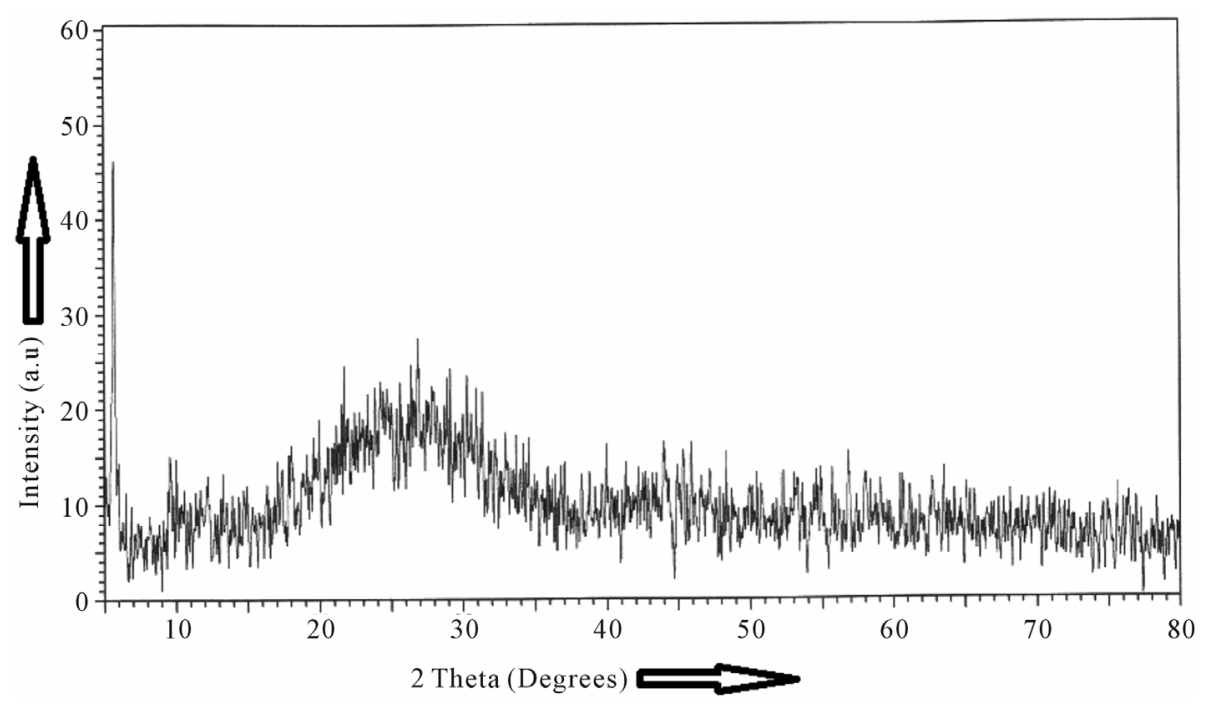

(a)

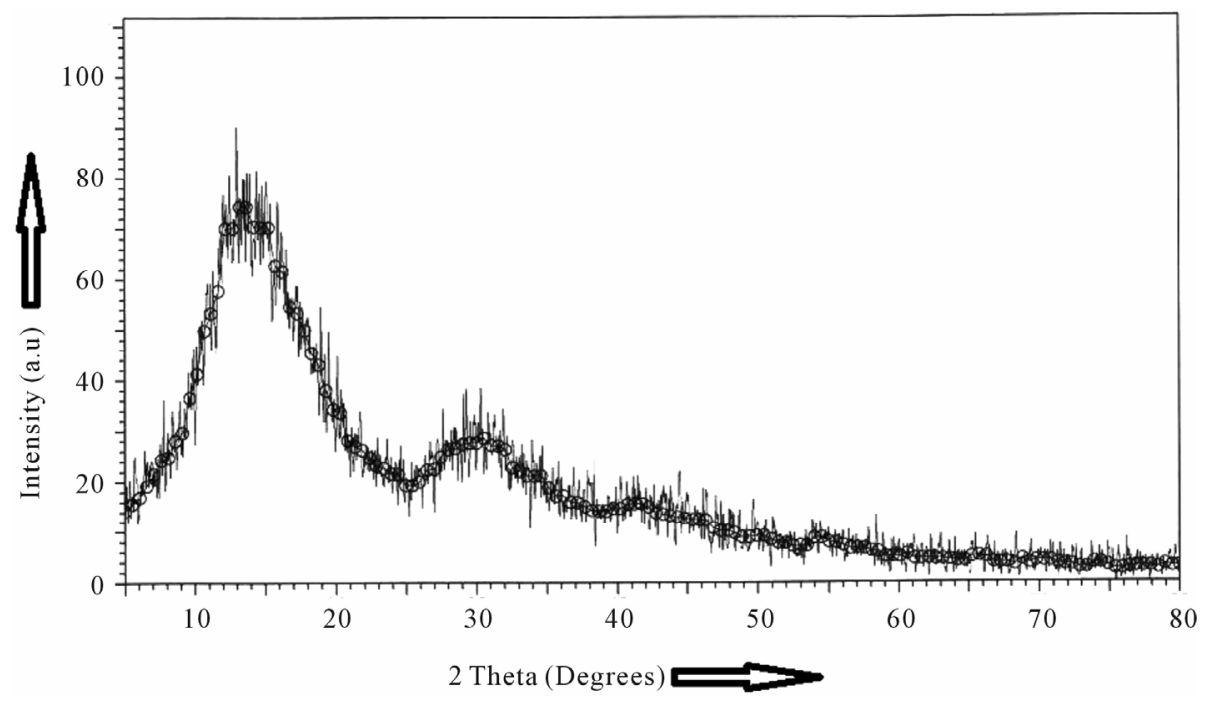

(b)

Figure 1. X-ray diffractograms of (a) glass powder, (b) glass ionomer cement after setting for 48 hours.

amorphous materials. The X-ray diffractograms of the powdered GIC pellets are indicative of an amorphous structure with pronounced radial distribution which results probably due to the complex form of the polymeric carboxylic acid with the glass powder.

Figure 2 shows the DC electrical conductivity of the sample. It can be seen from the figure that the electrical conductivity decreases as the temperature increases from $300 \mathrm{~K}$ to $353 \mathrm{~K}$ (i.e. $80^{\circ} \mathrm{C}$ ) and there after remains constant. The conductivity remains almost the same when the sample is subjected to further thermal cycling.

The dielectric constant and loss factor as a function of frequency are shown in Figure 3. The sample exhibits high dielectric constant at lower frequencies when compared to samples thermally annealed at $80^{\circ} \mathrm{C}$ for ten minutes. This difference can be attributed to the space charge polarization caused by mobile charge carriers which are present in the prepared samples but are absent in the samples annealed at $80^{\circ} \mathrm{C}$. Similarly the prepared sample has a characteristic loss peak below $0.1 \mathrm{MHz}$ which is absent in the sample annealed at $353 \mathrm{~K}$.

The DSC and TGA thermograms are shown in Figure 4. The DSC thermogram shows an exothermic peak at $100^{\circ} \mathrm{C}$ while the TGA thermogram indicates a simultaneous weight loss at the same temperature. Generally the loss of waters of hydration must be accompanied by an endothermic peak. But interestingly there is an exothermic peak at $100^{\circ} \mathrm{C}$ which can be attributed to loss of waters of hydration accompanied by a secondary setting reaction in which the heat evolved is greater than the 


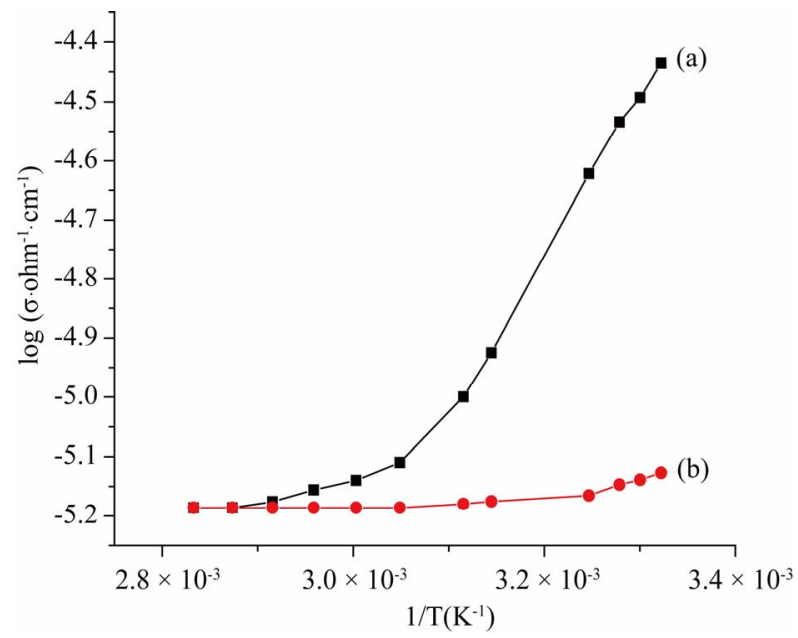

Figure 2. Electrical conductivity of (a) as prepared sample, (b) the sample annealed at $353 \mathrm{~K}$.

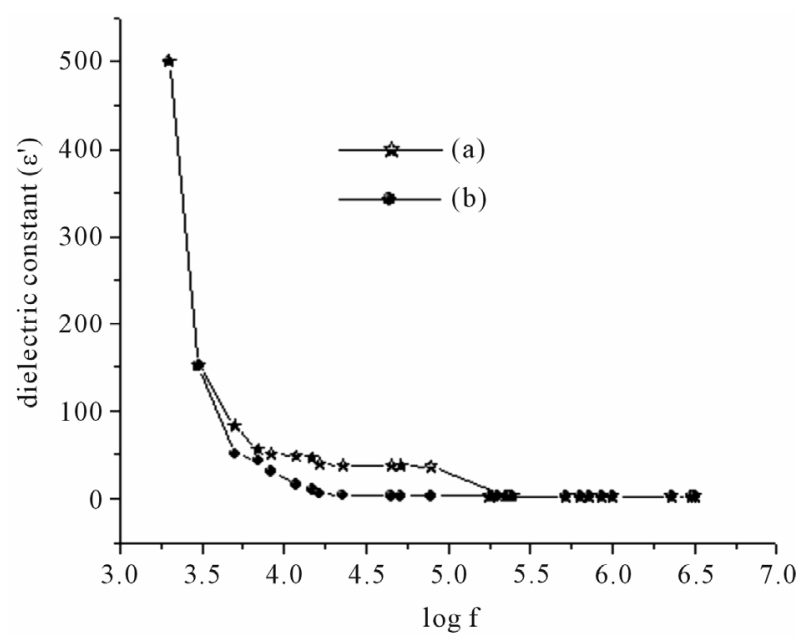

(A)

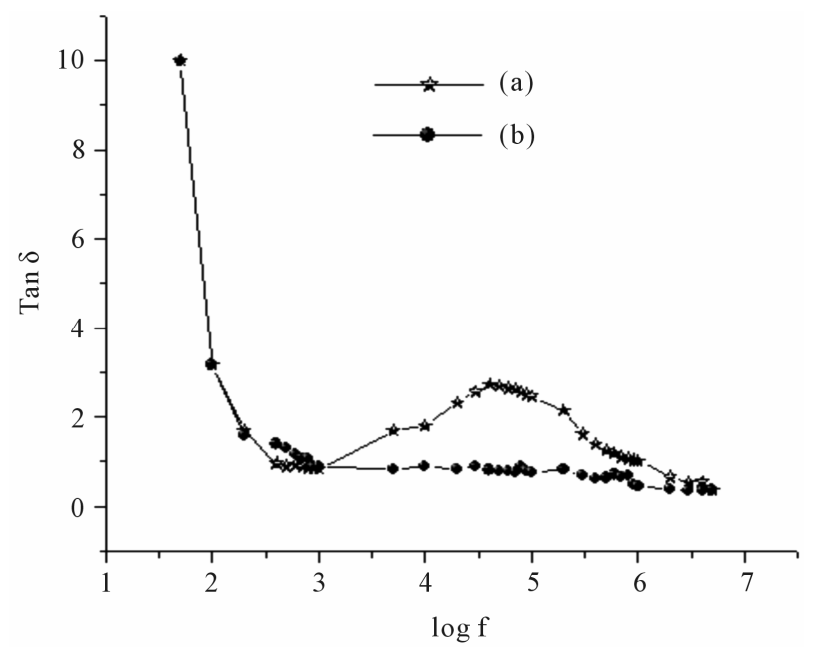

(B)

Figure 3. Frequency dependent (A) dielectric constant ( $\left.\varepsilon^{\prime}\right)$ and (B) loss factor, (Tan $\delta$ ) of GIC pellets. (a) After setting for 48 hours, (b) after thermally annealing at $80^{\circ} \mathrm{C}$ for $10 \mathrm{~min}$. heat absorbed by the waters of hydration escaping from the cement matrix. Besides this DSC thermograms show endothermic peaks between $425^{\circ} \mathrm{C}$ to $600^{\circ} \mathrm{C}$ with simultaneous weight loss as can be seen in the TGA curves. These endothermic peaks can be attributed to the decomposing of carboxylic acid groups in the cement matrix. The GICs contain a hydrated polycarboxylate metal matrix with in which the alumino-silicate glass particles are bound [3]. Once the glass powder is mixed with aqueous polycarboxylic acid a reaction between the alkaline glass powder and the unsaturated acid gives the formation of a salt gel. The acid base reaction proceeding in the salt gel leads to the formation of bonding matrix in which water serves as a reaction medium and also as an essential component of the salt gel containing metal carboxylate complexes formed in the reaction. This can be considered as a primary reaction [10].

One of the reaction mechanisms is fluoride release. Systematic study of fluoride release has indicated that irrespective of the make all GIC materials released maximum fluoride ion in the first 24 hours, followed by a drop and stabilization with gradual release [11]. This gives rise to great ionic movement like the fluoride ion [12].

DC resistivity and dielectric measurements of GIC were reported earlier [8]. These studies indicate that resistivity vs. temperature increases and dielectric constant vs. frequency decreases gradually with increase in setting time. The trend seems to be the same irrespective of the make and the powder to liquid ratio. These studies indicate that the cements are highly ionic and polar and more conductive than other cements.

The X-ray diffractograms of some commercial GICs were reported by De Mayer et al. [12]. The broad peaks which are characteristic of amorphous material also show some super-imposed sharp peaks which can be attributed to the presence of $\mathrm{Fe}_{3} \mathrm{O}_{4}$ and $\mathrm{CaF}_{2}$. The present GIC also shows similar X-ray diffractogram but without any superimposed sharp peaks. DSC studies were reported for six commercial GICs keeping the samples at a constant temperature of $37^{\circ} \mathrm{C}$ and measuring the heat flow as a function of temperature [13]. Their DSC results show that all materials undergo an exothermic setting process and after the setting is over no more exothermic or endothermic peaks as a function of time were found [14, 15]. DSC thermograms were also reported for resin modified glass ionomers (RMGIC) under isothermal dynamic condition using visible light for curing the RMGICs [15]. We have not found in literature DSC/ DTA studies of GICs which indicate the exothermic peak at $100^{\circ} \mathrm{C}$. Even though we have not attempted to calculate the enthalpies, a systematic study by DSC/TGA of different commercial dental GICs will give interesting information about the enthalpies of the secondary setting 


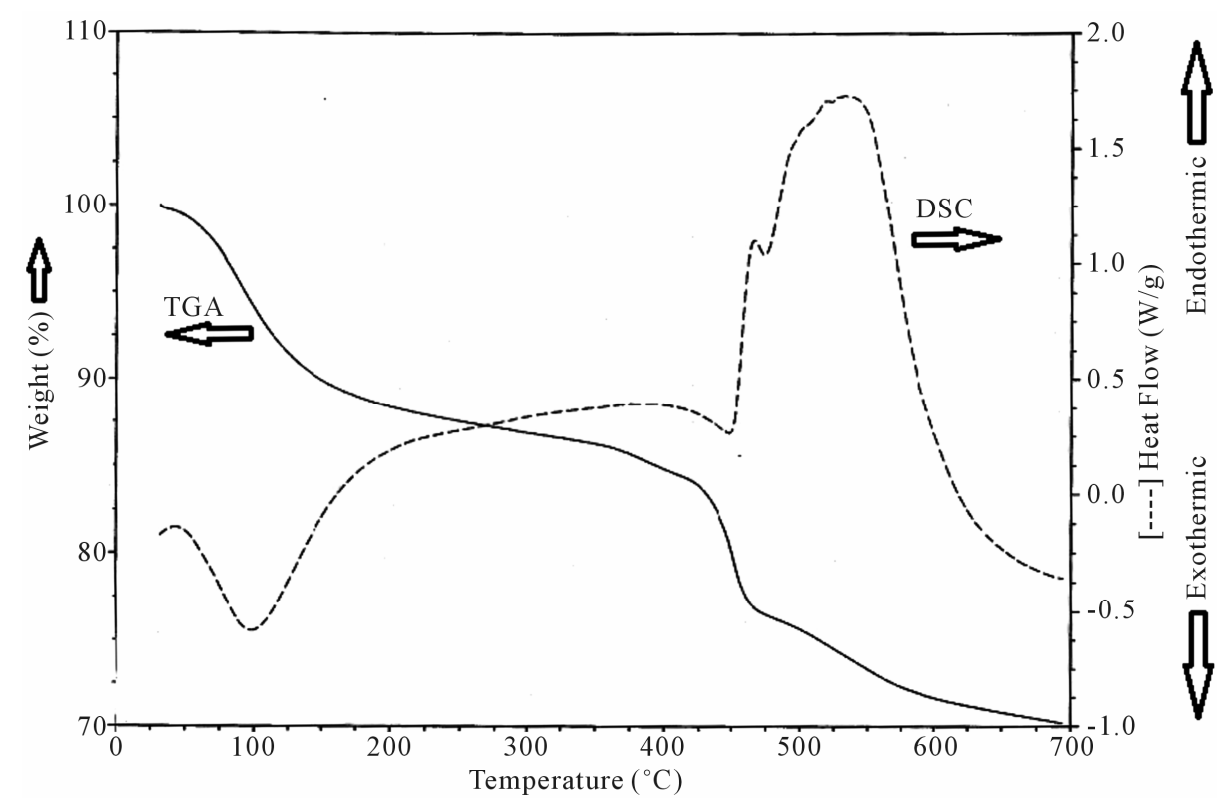

Figure 4. DSC and TGA thermograms for glass ionomer cement after setting for 48 hours.

reactions in these materials.

\section{CONCLUSION}

The X-ray studies indicate that the prepared samples of GIC are of highly disordered amorphous materials. The prepared sample exhibits high dielectric constant at lower frequencies when compared to thermally annealed samples. This can be attributed to the existence of space charge polarization in the prepared samples. But interestingly there is an exothermic peak at $100^{\circ} \mathrm{C}$ which can be attributed to loss of waters of hydration accompanied by a secondary setting reaction. DC electrical conductivity studies indicate that resistivity and dielectric constant increases with increase of setting time. This study also indicates that these GIC are ionic, polar and more conductive than other ones.

\section{REFERENCES}

[1] Wilson, A.D. and Nicholson, J.W. (1993) Acid-base cements: Their biomedical and industrial applications. Cambridge University Press, Cambridge. doi:10.1017/CBO9780511524813

[2] Milne, K.A., Calosi, N.J., O’Donnel, J.H., Kennard, C.H.L., Vega, S. and Marks, D. (1997) Glass ionomer dental restorative. Journal of Materials Science, 8, 349-356. doi:10.1023/A:1018576715479

[3] De Maeyer, E.A.P., et al. (1999) Stoichiometry of the leaching process of fluoride-containing aluminosilicate glass ionomer glasses. Journal of Dental Research, 78, 1312-1318. doi:10.1177/00220345990780070501

[4] Barry, T.I., Clinton, D.J. and Wilson, A.D. (1979) The structure of a glass ionomer cement and its relationship to the setting process. Journal of Dental Research, 58, 1072-1079. doi:10.1177/00220345790580030801

[5] De Maeyer, E.A.P., Verbeeck, R.M.H. and Vercruysse, C.W.J. (2002) Infrared spectrometric study of acid-degradable glasses. Journal of Dental Research, 81, 552555. doi:10.1177/154405910208100810

[6] Young, A.M., Sherpa, A., Pearson, G., Schottlander, B. and Waters, D.N. (2000) Use of Raman spectroscopy in the characterisation of the acid-base reaction in glassionomer cements; Biomaterials, 21, 1971-1979.

[7] Stamboulis, A., Matsuya, S., Hill, R.G., Law, R.V., Udoh, K., Nakagawa, M. and Matsuya, Y. (2006) MAS-NMR spectroscopy studies in the setting reaction of glass ionomer cements. Journal of Dentistry, 34, 574-581. doi:10.1016/j.jdent.2005.08.006

[8] Tay, W.M. and Braden, M. (1984) Materials science dielectric properties of glass-ionomer cements-further studies. Journal of Dental Research, 63, 74-75. doi:10.1177/00220345840630011901

[9] Tay, W.M. and Braden, M. (1987) Thermal diffusivity of glass ionomer cements. Journal of Dental Research, 66, 1040-1043. doi:10.1177/00220345870660051001

[10] Eden, O.R., Foster, G.M. and Hooper, R.M. (2003) Investigation of the mechanical performance of young glass ionomer cement using dynamic mechanical analysis. Journal of Materials Science: Materials in Medicine, 14, 373378.

[11] DeMaeyer, E.A.P., Verbeeck, R.M.H. and Vercruysse, C.W.J. (1999) Stoichiometry of the leaching process of fluoride-containing aluminosilicate glass ionomer glasses. Journal of Dental Research, 78, 1312-1318. doi:10.1177/00220345990780070501

[12] De Maeyer, E.A.P. and Verbeeck, R.M.H. (2001) X-ray diffraction study of acid-degradable glasses. Journal of Dental Research, 80, 1764-1767. 
doi:10.1177/00220345010800081601

[13] Khalil, S.K.H. and Atkins, E.D.T. (1998) Investigation of glass ionomer cements using differential scanning calorimetry. Journal of Materials Science: Materials in Medicine, 9, 529-533. doi:10.1023/A:1008892006086

[14] Berzins, D.W., Abey, S., Costache, M.C., Wilkie, C.A. and Roberts, H.W. (2010) Resin-modified glass ionomer setting reaction competition. Journal of Dental Research, 89, 82-86. doi:10.1177/0022034509355919

[15] Kartelia, E.M. (2011) Thermal characterization of magnesium containing ionomer glasses. Ph.D Thesis, University of Birmingham, Birmingham. 